\title{
Using exome sequencing to identify the cause of myocardial hypertrophy in a Chinese family
}

\author{
TIAN PU ${ }^{1}$, QIANQIAN GUO ${ }^{1}$, RUIXUE CAO ${ }^{1}$, RANG XU $^{2}$, KUN SUN $^{1}$ and SUN CHEN $^{1}$ \\ ${ }^{1}$ Department of Pediatric Cardiology; ${ }^{2}$ Scientific Research Center, Xinhua Hospital, \\ Shanghai Jiaotong University School of Medicine, Shanghai 200092, P.R. China
}

Received July 30, 2014; Accepted April 15, 2015

DOI: $10.3892 / \mathrm{mmr} .2015 .3818$

\begin{abstract}
Myocardial hypertrophy is a common feature of numerous diseases. It is important to distinguish between these diseases in order to enable accurate diagnosis and the administration of appropriate therapy. Using whole-exome sequencing, the present study aimed to identify a pathogenic mutation in a Chinese family, which may lead to cardiac hypertrophy and Wolff-Parkinson-White syndrome. The proband from the Chinese family exhibited left ventricular hypertrophy and pre-excitation with a short PR interval. DNA was extracted from peripheral blood obtained from the subject family, and exome sequencing was performed in the proband. Polymerase chain reaction and direct sequencing were used to confirm the presence of a mutation, and confirmed that the pathogenic mutation was 5'-AMP-activated protein kinase subunit $\gamma 2$ (PRKAG2) (p.R302Q), which has been previously reported in a family with an inherited from of WPW. A stop-gain mutation in urotensin II receptor (UTS2R) (p.S241X), which is associated with congestive heart failure, was identified in the proband and in one other affected family member. It is important to identify the causes of myocardial hypertrophy, in order to provide a theoretical basis with which to improve clinical diagnosis and the assessment of prognosis. The results of the present study suggest that if a patient has myocardial hypertrophy with a short PR interval on electrocardiogram, a mutation in the PRKAG2 gene should be considered. In conclusion, exome sequencing methods may assist with the identification of causative genes in myocardial hypertrophy, as well as genes that are associated with an increased risk of sudden cardiac death.
\end{abstract}

Correspondence to: Dr Sun Chen, Department of Pediatric Cardiology, Xinhua Hospital, Shanghai Jiaotong University School of Medicine, 1665 Kongjiang Road, Shanghai 200092, P.R. China E-mail: chensundoc@163.com

Key words: myocardial hypertrophy, whole-exome sequencing, Wolff-Parkinson-White syndrome, 5'-AMP-activated protein kinase subunit $\gamma^{2}$

\section{Introduction}

Myocardial hypertrophy is characterized by an increased thickness of the heart wall, and is a common clinical manifestation of a number of hereditary disorders. During childhood, several diseases may lead to a phenotype mimicking cardiac hypertrophy (1). The mitochondrial syndromes MELAS, MERRF and Kearns-Sayre syndrome affect multiple mitochondrial proteins. If gene defects occur in respiratory chain enzymes, it usually takes the form of hypertrophic cardiomyopathy (HCM) (2). The majority of instances of myocardial hypertrophy are caused by HCM, which is an autosomal dominant disease that is predominantly characterized by ventricular hypertrophy, including hypertrophy of the interventricular septum, and atrial enlargement. Mutations are detected in the sarcomere protein in $60 \%$ of patients with $\mathrm{HCM}$, which is a functional unit required for the contraction of myocytes $(3,4)$. However, $<10 \%$ of cases of cardiac hypertrophy are caused by other diseases, including aortic valve disease, Fabry's disease (5), Danon disease, Pompe disease, Noonan syndrome (6) and hypertension (1).

Although HCM and other diseases may all result in myocardial hypertrophy, genetic studies have revealed that the pathogenic genes and mutations differ among these conditions (7). To date, $>630$ mutations in $10 \mathrm{HCM}$ susceptibility genes have been identified. These genes encode sarcomeric proteins, including $\beta$-myosin heavy chain ( $\beta$ MHC), regulatory myosin light chain, essential myosin light chain, $\beta$-cardiac myosin binding protein $\mathrm{C}$, cardiac troponin $\mathrm{T}$, cardiac troponin I, $\alpha$-tropomyosin and cardiac actin (8). The $\beta$ MHC gene was the first to be identified as a cause of familial hypertrophic cardiomyopathy (9). Subsequently, 100 disease-associated mutations have been defined, including myosin heavy chain 7 (MYH7), myosin light chain 3 (MYL3), MYL2, actin $\alpha$ cardiac muscle 1 (ACTC1), tropomyosin 1 (TPM1), troponin T type 2 (TNNT2), troponin I type 3 (TNNI3), troponin C type 1 (TNNC1), cardiac myosin -binding protein C (MYBPC3), actinin $\alpha 2$ (ACTN2), titin (TTN) and 5'-AMP-activated protein kinase subunit $\gamma 2$ (PRKAG2) (10). Furthermore, other candidate genes, including acid $\alpha$-glucosidase (GAA) (11), bone morphogenetic protein 2 (BMP2) (12), NK2 homeobox 5 (NKX2.5) (13), lysosome-associated membrane protein 2 (LAMP-2) (14), tyrosine-protein phosphatase non-receptor type 11 (PTPN11) (15), son of sevenless homolog 1 (SOS1) (16) 
and V-Ki-ras2 Kirsten rat sarcoma viral oncogene homolog (KRAS) (17), have a close association with metabolic disease and malformation syndrome.

It is important to identify the causes of myocardial hypertrophy, in order to provide a theoretical basis for improved clinical diagnosis and assessment of prognosis. New generation sequencing technologies have been employed to detect the genetic causes of various human diseases. Exome sequencing is a high throughput sequencing technology, which focuses on exonic regions, which harbor $85 \%$ of mutations and exert large effects on disease-associated traits in the human genome $(18,19)$. Therefore, exome sequencing may be a better choice with which to identify mutations involved in human diseases than Genome wide association studies and Sanger sequencing. Furthermore, exome sequencing may be used to identify the cause of several Mendelian-inherited diseases and to identify genes associated with an increased risk of sudden cardiac death (SCD).

The present study aimed to identify causes of cardiac hypertrophy. A 10-year-old boy exhibiting left ventricular hypertrophy (LVH) and pre-excitation with a short PR interval, was selected as the proband of a Chinese family. Exome sequencing was used to identify the mutation responsible. The results suggested that it is important to confirm the genetic causes of cardiac hypertrophy in order to improve the diagnosis and treatment of this disease.

\section{Materials and methods}

Clinical evaluation. Surviving members of the family $(n=22)$ were assessed by a detailed analysis of their medical history, a physical examination, 12-lead electrocardiography (ECG) and two-dimensional echocardiography (Fig. 1). Ventricular pre-excitation was diagnosed on the basis of a short PR interval ( $<120 \mathrm{~ms})$, a widened QRS interval ( $>110 \mathrm{~ms})$ and an abnormal initial QRS vector ( $\delta$ wave). When the thickness of the left ventricular free wall or the ventricular septum was $>13 \mathrm{~mm}$, left ventricular hypertrophy was diagnosed. Written, informed consent was obtained from all participants, according to the guidelines of the Ethics committee of Xinhua Hospital (Shanghai, China). Clinicians and nurses undertook the clinical assessments for the proband and their family in the Department of Pediatric Cardiology, XinHua Hospital.

Exome capture sequencing. Blood samples were obtained from each individual and DNA was extracted from whole blood using the QIAamp DNA blood mini kit.(Qiagen $\mathrm{GmbH}$, Hilden, Germany) according to the manufacturer's instructions. Whole-exome sequencing was performed in the proband. The extracted genomic DNA was sonicated in order to produce fragments and hybridized to the capture array, according to the manufacturer's instructions. Using the NimbleGen linkers added to the exonic DNA fragments, the enriched DNA fragments were eluted and amplified using ligation-mediated polymerase chain reaction (PCR). The degree of enrichment of the exonic sequences required quantitative PCR for estimation prior to the second run of library construction. A minimum requirement of an 80-fold enrichment was prepared for the following procedure. The enriched exonic DNA was ligated with DNA ligase to fragments ranging in size between 2 and
$5 \mathrm{~kb}$ randomly. The resultant DNA fragments were cut into $200 \mathrm{bp}$, on average, and were submitted to standard Illumina Hiseq 2000 library preparation.

The proband DNA was sent to a commercial provider (Shanghai Biotechnology Co, Ltd, Shanghai, China), which performed sequencing using the Hiseq 2000 platform. Image analysis and base calling were performed with the Illumina's Consensus Assessment of Sequence and Variation 1.8, using the default parameters. Following removal of reads that contained sequencing adaptors, and low-quality reads with $>5$ unknown bases, high quality reads were aligned to the NCBI human reference genome (hg19 build) using the Burrows-Wheeler Aligner (BWA) version 0.6.2 (20). Post-alignment procedure and duplicate removal was performed using SAM tools version 0.1.18 and Picard version 1.49 (21). Local realignment of the BWA-aligned and base recalibration were performed using the Genome Analysis Tool kit.

Mutation detection and validation. All changes were filtered against exome data from ethnic Han Chinese individuals in the 1,000 Genomes Project (http://www.1000genomes.org/) and against the Han Chinese small nucleotide polymorphisms in the dbSNP135 (http://ncbi.nlm.nih.gov/). In order to confirm the candidate variants, Sanger sequencing was performed on an ABI 3730 capillary sequencing instrument (Applied Biosystems, Foster City, CA, USA). Primers for exon 3 of PRKAG2 (NM_02449) and exon 1 of urotensin II receptor (UTS2R; NM_018949) were designed for polymerase chain reaction amplification (Sangon Biotech Co., Ltd., Shanghai, China). The sequences of these primers are shown in Table I. The PCR cycling conditions were as follows: $98^{\circ} \mathrm{C}$ for $1 \mathrm{~min}$, 35 cycles of $98^{\circ} \mathrm{C}$ for $20 \mathrm{sec}, 56^{\circ} \mathrm{C} / 68^{\circ} \mathrm{C}$ for $30 \mathrm{sec}$ and $72^{\circ} \mathrm{C}$ for $30 \mathrm{sec}$, then $72^{\circ} \mathrm{C}$ for $10 \mathrm{~min}$ and $4^{\circ} \mathrm{C}$ until use.

\section{Results}

Clinical evaluation. Affected individuals $(n=6)$ from a four-generation family were diagnosed with Wolff-Parkinson-White (WPW) syndrome. Their 12-lead ECGs provided evidence of ventricular pre-excitation. The patients also presented with dyspnea, palpitations, syncope and fatigue symptoms (Table II). Patient III-5 was diagnosed with WPW and died suddenly at age 28. In addition to pre-excitation, two females (Patient II-4 and III-3) had resting heart rates of $<50$ beats per minute. Patient II- 4 received a permanent pacemaker implant and succumbed to lung cancer at age 61. Patient III-3 exhibited syncope on one occasion. Cardiac hypertrophy was identified in 2 of the 6 affected subjects (33\%). However, subject III-6 manifested no clinical symptoms. As a result of the mortality of the first generation, it is unknown whether these patients exhibited WPW or any other phenotype.

Identification of PRKAG2 mutations through exome sequencing. Whole-exome sequencing of genomic DNA obtained from the peripheral blood of the proband was performed. There were 126, 101 and 848 total reads produced by exome sequencing. Following elimination of PCR repetition, the number of unique mapped reads was 110,840 and 477 , which held $89.02 \%$ of the mapped reads. The coverage 
Table I. Oligonucleotide primer sequences used for gene amplification.

\begin{tabular}{|c|c|c|c|c|}
\hline Variant & Orientation & Sequence $\left(5^{\prime}-3^{\prime}\right)$ & Fragment size (bp) & $\operatorname{Tm}\left({ }^{\circ} \mathrm{C}\right)$ \\
\hline \multirow[t]{2}{*}{ PRKAG2_rs121908987 } & Forward & ATTTCTAATCCCTGTATGCC & 718 & 56 \\
\hline & Reverse & ACCCTGCCAGCAAGAATG & & \\
\hline \multirow[t]{2}{*}{ UTS2R/exon1 } & Forward & CCCAAGGGCTACCGCAAG & 343 & 68 \\
\hline & Reverse & GCCAGAAGGGCAGGAAGCAG & & \\
\hline \multirow[t]{2}{*}{ TTN/exon 15} & Forward & AGGCATCAATAGCCGGTAG & 399 & 54 \\
\hline & Reverse & TATGGCAAAGGAGAAAGG & & \\
\hline \multirow[t]{2}{*}{ TNN/exon149 } & Forward & CCGAAAATAAATATGGTG & 331 & 52 \\
\hline & Reverse & AACTGTTCTCAGGGAAAT & & \\
\hline
\end{tabular}

Tm, melting temperature.

Table II. Clinical characteristics of affected individuals.

\begin{tabular}{lcccc}
\hline ID number & Age/sex & ECG & LVWT mm & Clinical \\
\hline II-4 & $61 / \mathrm{F}$ & PR & N/A & C, P, V \\
III-2 & $36 / \mathrm{M}$ & PR, LVH & 11.7 & $\mathrm{C}, \mathrm{P}, \mathrm{D}$ \\
III-3 & $37 / \mathrm{F}$ & PR, LVH, RBBB & 18.2 & P, S \\
III-5 & 28/M & PR & N/A & SCD \\
III-6 & PR & 10 & A \\
IV-1 & PR & PR & 14.7 & C, P \\
\hline
\end{tabular}

ECG, electrocardiogram; F, female; M, male; PR, short PR (<100 ms); LVH, left ventricular hypertrophy; RBBB, right bundle branch block; LVWT, maximum left ventricular wall thickness; N/A, not available; C, chest pain; P, palpitations; V, permanent pacemaker inserted; D, dyspnea; S, syncope; SCD, sudden cardiac death; A, asymptomatic.

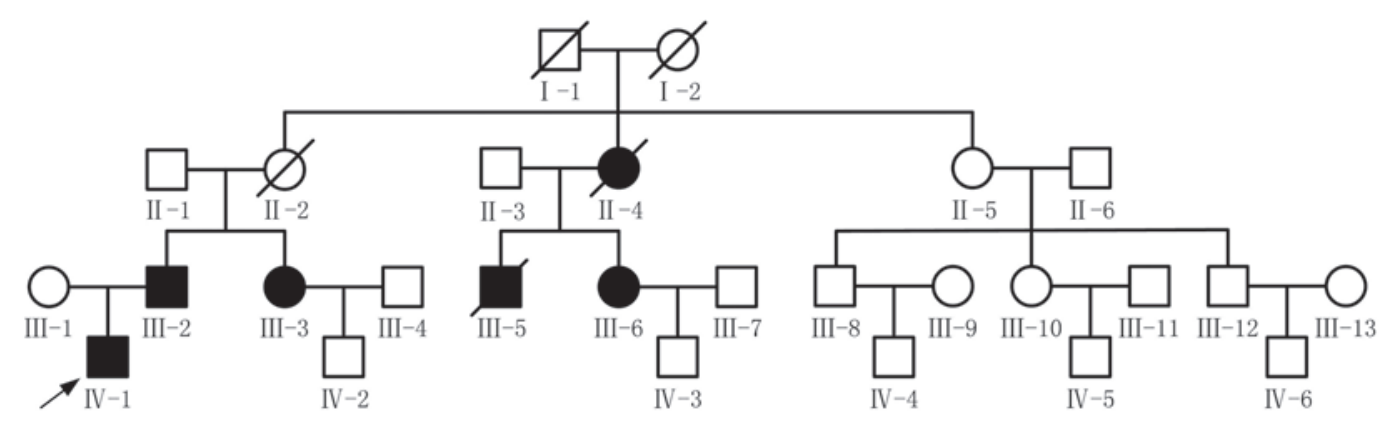

Figure 1. Pedigree of a family, in which some members exhibited myocardial hypertrophy and Wolff-Parkinson-White syndrome. IV-1 was the proband (arrow). All members were screened, II-4 and II-5 died during the study, while I-1, I-2 and II-2 had died prior to the study.

of $1 \mathrm{x}, 10 \mathrm{x}$ and $50 \mathrm{x}$ was measured in the capturing target area, accounting for $99.96,99.4$ and $88.92 \%$, respectively. Finally, 20 and 449 exonic variations were identified in the protein-coding region. According to the screening criteria, 85 variants, including 84 non-synonymous single nucleotide variants (SNVs) and 1 stop-gain SNV (UTS2R), were identified. However, none of these SNVs revealed any association with cardiac hypertrophy or WPW, while UTS2R (p.S241X) appeared to be closely associated with heart failure.

According to OMIM (http://www.ncbi.nlm.nih.gov/omim), a list of genes associated with cardiac hypertrophy and WPW syndrome was identified, which contained MYH7, MYL3,
MYL2, ACTC, TPM1, TNNT2, TNNI3, TNNC1, MYBPC3, ACTN2, TTN, PRKAG2, GAA, LAMP-2, PTPN11, SOS1, KRAS, RAF1 and NKX2.5. A comparison between these candidate genes and the whole-exome sequencing of the propositus, identified mutations in PRKAG2 (p.R302Q), TTN (p.D20198E), TTN (p.K811T) and UTS2R (p.S241X). Since PRKAG2 (p.R302Q) has been previously reported, the present study aimed to confirm whether TTN and UTS2R were polymorphic in the affected individuals in this family. Normal DNA samples $(n=100)$ were sequenced to confirm whether UTS2R (p.S241X), TTN (p.D20198E) and TTN (p.K811T) were polymorphisms. Family members of the patients III-2, 
A

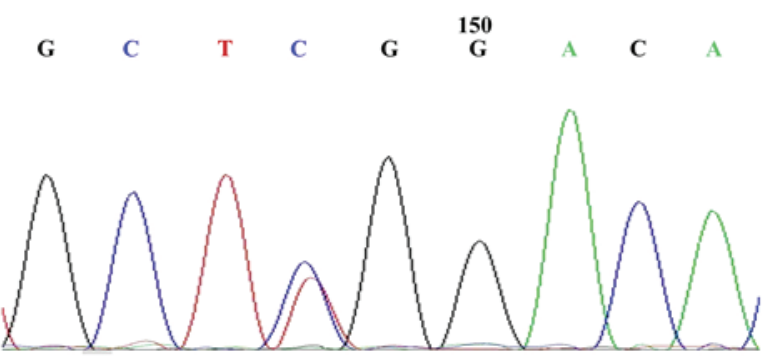

B
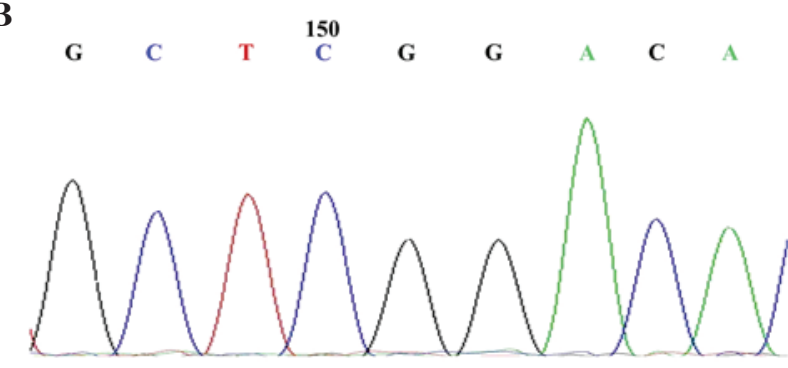

C
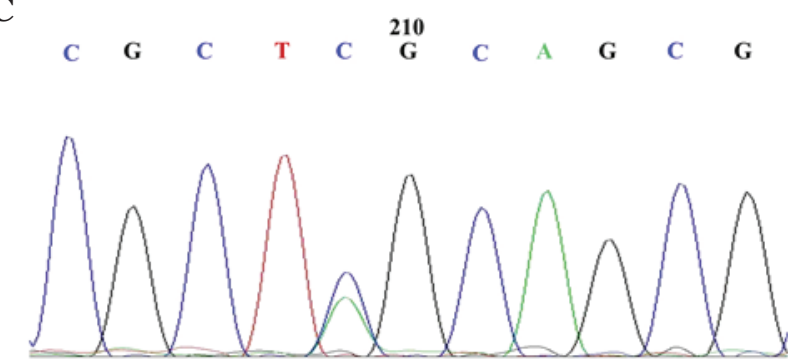

D

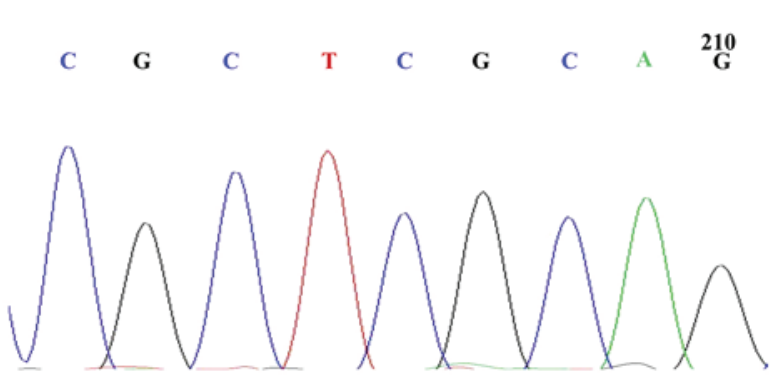

Figure 2. Sequence analysis of the PRKAG2 mutation. (A) Sequence analysis results demonstrated mutation PRKAG2 (p.R302Q) in the proband. (B) Sequence analysis results demonstrated the normal sequence. (C) Sequence analysis results demonstrated mutation UTS2R (p.S241X) of the proband; (D) Sequence analysis results demonstrated the normal sequence.

III-3, III-6, IV-1, II-4, III-5 and IV-3 were shown to exhibit the PRKAG2 mutation, while others revealed no mutation, with the exception of the members who had died, I-1 and I-2 (Fig. 2).

\section{Discussion}

In the present study of one family, in which the proband exhibited cardiac hypertrophy and WPW syndrome, 6/25 family members, excluding the first generation, were found to be affected. All affected members exhibited electrocardiographic evidence of pre-excitation. The patients also exhibited clinical symptoms, including dyspnea, palpitations, syncope and fatigue. A previous study demonstrated that WPW is likely to be responsible for $10.5 \%$ of cases of SCD in affected individuals $<35$ years (22). The risk of SCD for patients with
WPW syndrome is $0.02 \%$ per year (23). Only one SCD had occurred in the family involved in the present study, in which the incidence of SCD was therefore $16.6 \%$, which is marginally higher than $0.02 \%$ (22). Although the prevalence of SCD appears to have improved, no clear difference was shown, due to the limited number of cases examined.

The results were in accordance with the results of a study by Gollb et al (24), which first demonstrated mutations in the PRKAG2 gene in a family with an inherited form of WPW, who presented with cardiac hypertrophy, ventricular pre-excitation and AV nodal disease. PRKAG2 encodes the regulatory subunit of AMP-activated protein kinase, and >11 PRKAG2 mutations have been identified, including a frame-shift mutation (L351Ins) and 10 missense mutations (R302Q, H383R, T400 N, Y487H, N488I, E506 K, R531G, R531Q, S548P and G100S) (25). The cardiac phenotype of the PRKAG2 mutation is similar to that of glycogen storage cardiomyopathy and HCM. Therefore, other genes associated with these conditions may also be candidates for causing myocardial hypertrophy, including NKX2.5, BMP2, MYH7, MYL3, MYL2, ACTC1, TPM1, TNNT2, TNNI3, TNNC1, MYBPC3, ACTN2, TTN, PRKAG2, LAMP-2 and GAA. Taking these factors into account, the present study performed whole-exome sequencing of the proband and analyzed genes, which were known to be associated with cardiac hypertrophy and WPW. PRKAG2 (p.R302Q) was found to be the causative mutation in the affected individuals and a search for mutations within the other candidate genes failed to reveal sequence alternations. An identical mutation was identified in all affected members and was absent in all unaffected members of the family, with the exception of patient IV-3. Sidhu et al (26) created PRKAG2 transgenic mice model in order to identify the conduction accessory pathway, and Tan et al (27) reported that ventricular pre-excitation, caused by PRKAG2 (p.R302Q), was associated with Mahaim fibers. In the present study, it was observed that patient IV-3 (12-years old) expressed the identical mutation, whilst remaining asymptomatic. Penetrance may be affected by mutation-specific phenotypes resulting from the patient's own genetic and environmental background. As the gene carrier is at risk of developing WPW, they should be closely monitored in the future. The association between genotype and phenotype remains to be elucidated.

Notably, the current study identified a stop-gain mutation, termed UTS2R (p.S241X), in patients III-2 and IV-1, by Sanger sequencing. Human urotensin II has several cardiovascular effects. A previous study demonstrated marked expression of urotensin II in cardiomyocytes, with reduced expression in patients with early-stage congestive cardiac failure (28). The symptoms of the two members with this mutation, were more severe than those of other individuals in this family, and the two carriers may have a tendency to develop heart failure. Massive exome sequencing may provide a reliable basis for the identification of genes associated with an increased risk of cardiac events, although the financial cost of such an undertaking would be significant.

A number of patients manifest hypertrophic cardiomyopathy as an initial finding. The present study of a single family suggested that it may be beneficial to assess patient gene mutations, particularly in children whose echocardiography reveals 
cardiac hypertrophy, and who exhibit a short PR interval on ECG. Gene carriers who have no clinical manifestations are often encountered clinically. Exome sequencing methods may provide genetic evidence to identify pathogenic genes. In addition, the identification of relevant risk factors to avoid the occurrence of SCD is required. With the development of next generation sequencing technology, this approach may make medical genomics a reality.

\section{Acknowledgements}

This study was supported by a Project supported by the National Natural Science Foundation of China (grant no. 81070134/30772349), the National Basic Research Program of China (grant no. 2010CB529501), the Joint health research program for major diseases in Shanghai (grant no. 2013ZYJB0016) and the Shanghai Committee of Science and Technology China (grant no. 124119a3900). The authors would like to thank all the staff at Xinhua Hospital (Shanghai, China) for their assistance, and all the members of the family involved in this study.

\section{References}

1. Marin-Garcia J, Ananthakrishnan R, Goldenthal MJ, Filiano JJ and Perez-Atayde A: Cardiac mitochondrial dysfunction and DNA depletion in children with hypertrophic cardiomyopathy. J Inherit Metab Dis 20: 674-680, 1997.

2. Scaglia F, Towbin JA, Craigen WJ, et al: Clinical spectrum, morbidity, and mortality in 113 pediatric patients with mitochondrial disease. Pediatrics 114: 925-931, 2004.

3. Erdmann J, Daehmlow S, Wischke S, et al: Mutation spectrum in a large cohort of unrelated consecutive patients with hypertrophic cardiomyopathy. Clin Genet 64: 339-349, 2001.

4. Richard P, Charron P, Carrier L, et al: Distribution of disease genes in 102 genotyped families with hypertrophic cardiomyopathy. Circulation 104: 521-521, 2001.

5. Sachdev B, Takenaka T, Teraguchi H, Tei C, Lee P, McKenna WJ and Elliott PM: Prevalence of Anderson-Fabry disease in male patients with late onset hypertrophic cardiomyopathy Circulation 105: 1407-1411, 2002.

6. Patra S, Subramaniun A, Mahimaiha J, Sastry UM and Nanjappa MC: Apical hypertrophic cardiomyopathy in an infant: first presentation of pompe's disease. World J Pediatr Congenit Heart Surg 5: 491-493, 2014.

7. Moak JP and Kaski JP: Hypertrophic cardiomyopathy in children. Heart 98: 1044-1054, 2012.

8. Marian AF and Roberts R: The molecular genetic basis for hypertrophic cardiomyopathy. J Mol Cell Cardiol 33: 655-670, 2001.

9. Seidman CE, Seidman JG, InScriver CR, et al: (eds): The Metabolic and Molecular Basis of Inherited Disease. 433-5418, 2001.
10. Xu Q, Dewey S, Nguyen S and Gomes AV: Malignant and benign mutations in familial cardiomyopathies: Insights into mutations linked to complex cardiovascular phenotypes. J Mol Cell Cardiol 48: 899-909, 2010.

11. Howell RR, Byrne B, Darras BT, Kishnani P, Nicolino M and van der Ploeg A: Diagnostic challenges for Pompe disease: an under-recognized cause of floppy baby syndrome. Genet Med 8: 289-296, 2006.

12. Lalani SR, Thakuria JV, Cox GF, et al: 20p12.3 microdeletion predisposes to Wolff-Parkinson-White syndrome with variable neurocognitive deficits. J Med Genet 46: 168-175, 2009.

13. Jay PY, Harris BS, Maguire CT, et al: Nkx2-5 mutation causes anatomic hypoplasia of the cardiac conduction system. J Clin Invest 113: 1130-1137, 2004.

14. Nishino I, Fu J, Tanji K, et al: Primary LAMP-2 deficiency causes X-linked vacuolar cardiomyopathy and myopathy (Danon disease). Nature 406: 906-910, 2000.

15. Tartaglia M, Mehler EL, Goldberg R, et al: Mutations in PTPN11, encoding the protein tyrosine phosphatase SHP-2, cause Noonan syndrome. Nat Genet 29: 465-468, 2001.

16. Tartaglia M, Pennacchio LA, Zhao C, et al: Gain-of-function SOS1 mutations cause a distinctive form of Noonan syndrome. Nat Genet 39: 75-79, 2007.

17. Schubbert S, Zenker M, Rowe SL, et al: Germline KRAS mutations cause Noonan syndrome. Nat Genet 38: 331-336, 2006.

18. Metzker ML: Sequencing technologies-the next generation. Nat Rev Genet 11: 31-46, 2010.

19. Mamanova L, Coffey AJ, Scott CE, et al: Target-enrichment strategies for next-generation sequencing. Nat Methods 7: 111-118, 2010.

20. Liu Y, Schmidt B and Maskell DL: CUSHAW: a CUDA compatible short read aligner to large genomes based on the Burrows-Wheeler transform. Bioinformatics 28: 1830-1837, 2012.

21. Li H,Handsaker B, Wysoker A, et al: The sequence alignment/map format and SAMtools. Bioinformatics 25: 2078-2079, 2009.

22. Gollob MH, Green MS, Tang AS and Roberts R: PRKAG2 cardiac syndrome: Familial ventricular preexcitation, conduction system disease and cardiac hypertrophy. Curr Opin Cardiol 17: 229-234, 2002.

23. Fitzsimmons PJ, McWhirter PD, Peterson DW and Kruyer WB: The natural history of Wolff-Parkinson-White syndrome in 228 military aviators: A long-term follow-up of 22 years. Am Heart J 142: 530-536, 2001.

24. Gollb MH, Green MS, Tang AS, et al: Identification of a gene responsible for familial Wolff-Parkinson-White syndrome. N Engl J Med 344: 1823-1831, 2001.

25. Zhang BL, Ye Z, Xu RL, et al: Overexpression of G100S mutation in PRKAG2 causes Wolff-Parkinson-White syndrome in zebrafish. Clin Genet 86: 287-291, 2014.

26. Sidhu JS, Rajawat YS, Rami TG, et al: Transgenic mouse model of ventricular preexeitation and atrioventricular reentrant achycardia induced by an AMP-activated protein kinase loss-of-function mutation responsible for Wolff-Parkinson-White syndrome. Circulation 111: 21-29, 2005.

27. Tan HL, van der Wal AC, Campian ME, et al: Nodoventricular accessory pathways in PRKAG2-dependent familial preexcitation syndrome reveal a disorder in cardiac development. Circ Arrhythm Electrophysiot 1: 276-281, 2008.

28. Douglas SA, Tayara L, Ohlstein EH, Halawaa N and Giaid A: Congestive heart failure and expression of myocardial urotensin II. Lancet 359: 1990-1997, 2002. 